\title{
Pautas metodológicas según las bases de planes de estudio "E". Gestión de agencias de viajes.
}

Methodological guidelines according to the bases of study plans " $E$ ". Management of travel agencies.

MSc. Rebeca Olivera Elosegui. ${ }^{1}$, MSc. Maité Rodríguez González. ${ }^{2}$

\begin{abstract}
The Bachelor's Degree in Tourism is immersed in the process of assimilation of the "E" study plan, which has led the different groups that make up the disciplines to focus on adapting their subjects, and their contents, to the new ones trends and particularities of the new study plan. Such is the case of the Management of travel agencies, which is part of the base curriculum of the race and contributes to understanding the products "trips", essential theme to acquire a perception of the tourism phenomenon. That is why it is defined as the general objective of this research the application of the methodological guidelines according to the conceptual design bases of Plan "E" in the teaching-learning process of the subject Management of Travel Agency of the Degree in Tourism. Bibliographic analysis, documentary analysis, open interviews to professors and students and 2 questionnaires to students of the Bachelor of Tourism degree were applied as main methods, obtaining as main results the definition of the pertinent methodological guidelines for the subject and its impacts. in the teaching-learning process
\end{abstract}

Keywords: "E" Plan, Travel Agencies, Trends, Methodological Guidelines.

\section{Resumen}

La carrera de Licenciatura en Turismo se encuentra inmersa en el proceso de asimilación del plan de estudio "E", lo que ha propiciado que los diferentes colectivos que integran las disciplinas se enfoquen a adaptar sus asignaturas, y contenidos de las mismas, a las nuevas tendencias y particularidades del nuevo plan de estudio. Tal es el caso de la Gestión de agencias de viajes, la cual es parte del currículo base de la carrera y contribuye a comprender los productos "viajes", temática imprescindible para adquirir una percepción del fenómeno turístico. Es por ello que se define como objetivo general de la

\footnotetext{
${ }^{1}$ Máster en Marketing, Comunicación y Gestión Empresarial. Profesora Auxiliar de la Facultad de Turismo, Universidad de La Habana, Cuba. E-mail: rolivera@ ftur.uh.cu

${ }^{2}$ Máster en Gestión Turística. Profesora Auxiliar de la Facultad de Turismo, Universidad de La Habana, Cuba. E-Mail: mrodriguez@ftur.uh.cu
} 
presente investigación la aplicación de las pautas metodológicas según las bases conceptuales de diseño del Plan "E" en el proceso de enseñanza aprendizaje de la asignatura Gestión de Agencia de Viajes de la Licenciatura en Turismo. Se aplicaron como principales métodos el análisis bibliográfico, el análisis documental, las entrevistas abiertas a profesores y estudiantes y 2 cuestionarios a estudiantes de la carrera de Licenciatura en Turismo, obteniéndose como resultados primordiales la definición de las pautas metodológicas pertinentes para la asignatura y sus impactos en el proceso de enseñanza aprendizaje.

Palabras Clave: Plan “E”, Agencias De Viajes, Tendencias, Pautas Metodológicas.

\section{Introducción}

La Licenciatura de Turismo en Cuba, al igual del desarrollo del sector homónimo, resulta relativamente nueva en la Educación Superior; aspecto que ha marcado su evolución y adaptación a las necesidades prácticas del país, fundamentado en las experiencias propias y la transferencia de los centros de educación turística de referencia mundial.

Una aproximación general en este contexto, lo ha constituido el tránsito al llamado Plan "E", el cual desde su enfoque significa un reto a la preparación del docente, toda vez que rompe de alguna forma con métodos establecidos y desplegados durante la historia de la carrera.

De tal forma, el Plan "E" se orienta al aprendizaje significativo, al enfoque integrador y a la creación y desarrollo de competencias, modificando el rol del docente de centro a facilitador, de la reproducción a la creación, de la transmisión de conocimiento

s a la autogestión del conocimiento, apoyados por las novedades del entorno tecnológico, con énfasis en la información y la comunicación.

Las características de la agencia de viajes; núcleo de la asignatura del caso, exigen del profesional una visión integral de la actividad turística, así como una integración e interacción de todas las áreas de la gestión empresarial, a tenor de las particularidades de una empresa, definida por autores como fundamental para el turismo, en sus funciones de productor y distribuidor.

Los elementos anteriores indican la definición de un problema de carácter metodológico:

¿Cómo aplicar las bases conceptuales del Diseño de los Planes E de la Educación Superior cubana (Ministerio de Educación Superior), en la asignatura Gestión de Agencias de viajes de la Licenciatura en Turismo?

Se define como objetivo general: Aplicar las pautas metodológicas del proceso de enseñanza aprendizaje de la asignatura Gestión de agencia de viajes de la Licenciatura en Turismo, definidas a partir de las bases conceptuales del diseño del Plan "E".

Para el cumplimiento del mismo se aplicaron como principales métodos el análisis bibliográfico, el análisis documental, las entrevistas abiertas a profesores y estudiantes y cuestionarios a estudiantes de la carrera de Licenciatura en Turismo. 


\section{Desarrollo}

\section{Aplicación de las bases conceptuales del diseño del plan de estudio "e" en la asignatura gestión de agencias de viajes.}

El trabajo fue desarrollado en el Curso Diurno 2018/2019, en el año de liquidación del Plan D, a toda la matrícula de 4to año (92 estudiantes); lo que posibilitó el perfeccionamiento de la asignatura para su impartición en el Plan "E" en el segundo semestre del mismo curso.

Resulta interesante entonces, estructurar el presente trabajo a partir de las bases conceptuales anteriormente mencionadas, de forma tal que posibilite el cumplimiento del objetivo general.

1. Mayor nivel de esencialidad en los contenidos de las disciplinas.

2. La asignatura Gestión de Agencias de Viajes se inserta según el Plan "E" (y el D) en la disciplina Turismo y Viajes. Resulta significativo la contrastación de las funciones y operaciones de este tipo de empresa con las principales funciones profesionales, definidas por este Plan, que a continuación se detallan:

3. Preparar los contenidos de información sobre destinos y entidades a comunicar a los visitantes actuales y potenciales (tanto en emisores como en destinos).

4. Desarrollar los procesos de planificación, implementación y ejecución de la comercialización e intermediación de viajes (con formas tradicionales o mediante comercio electrónico.)

5. Realizar los procesos de asistencia y guía a los viajeros garantizando la comunicación y calidad de los servicios

6. Organizar y gestionar servicios de alojamiento, de alimentos y bebidas, de ocio y de enriquecimiento cualitativo personal, de transporte de pasajeros y de comercio de productos para el turismo (mayorista y minorista).

7. Participar con enfoque transdisciplinar, en los procesos de ordenación y planificación territorial del desarrollo turístico sostenible y en la gestión de destinos turísticos.

8. Aplicar herramientas de gestión basadas en los métodos y técnicas modernas relacionadas con los procesos empresariales y su vinculación con el entorno en el sector del turismo y la hospitalidad.

9. Diseñar y gestionar productos turísticos.

10. Diseñar, elaborar, evaluar y controlar proyectos de negocios para el desarrollo turístico con un enfoque integral.

11. Implementar las expresiones concretas de la cultura, la historia, la naturaleza y la sociedad cubanas en el proceso de gestión de la actividad de las unidades empresariales con enfoque sostenible.

12. Diseñar y ejecutar investigaciones que apoyen el proceso de toma de decisiones.

13. Trabajar en equipo (Ministerio de Educación Superior, 2016). 
De las funciones anteriormente expuestas y la propia actividad de las agencias de viajes, pueden establecerse tres grupos de las primeras:

A. Aplicables a cualquier entidad turística. (6, 8, 9, 10 y 11).

B. Directamente vinculadas a las funciones de las agencias de viajes.

i Función asesora (1)

ii Función mediadora (2 y 4 )

iii Función productora (3 y 7)

C. Participación indirecta de las agencias de viaje (5)

Estas relaciones exigen del trabajo metodológico de la asignatura con la orientación de la base conceptual de referencia, el estudio de planes y programas de las asignaturas de la carrera, establecido como objetivo en el Plan Metodológico de la disciplina para el curso 2018/2019 (Consolidar el carácter intra e interdisciplinar, a partir de métodos activos y problémicos de enseñanza).

Este estudio previo, posibilitó diferenciar los contenidos fundamentales de la asignatura, determinándose cuál es el aporte de cada una de las asignaturas precedentes (pertenecientes o no a la disciplina), para la comprensión de la misma, y cuál es el rol de la asignatura para la comprensión de las posteriores. Por otra parte, se revisó la división por temas de la asignatura, y se consideró en principio el mantenimiento de los definidos con anterioridad en el Plan "D”, excluyendo los contenidos de Gestión de Transporte Turístico, definida en el Plan "E "como asignatura independiente.

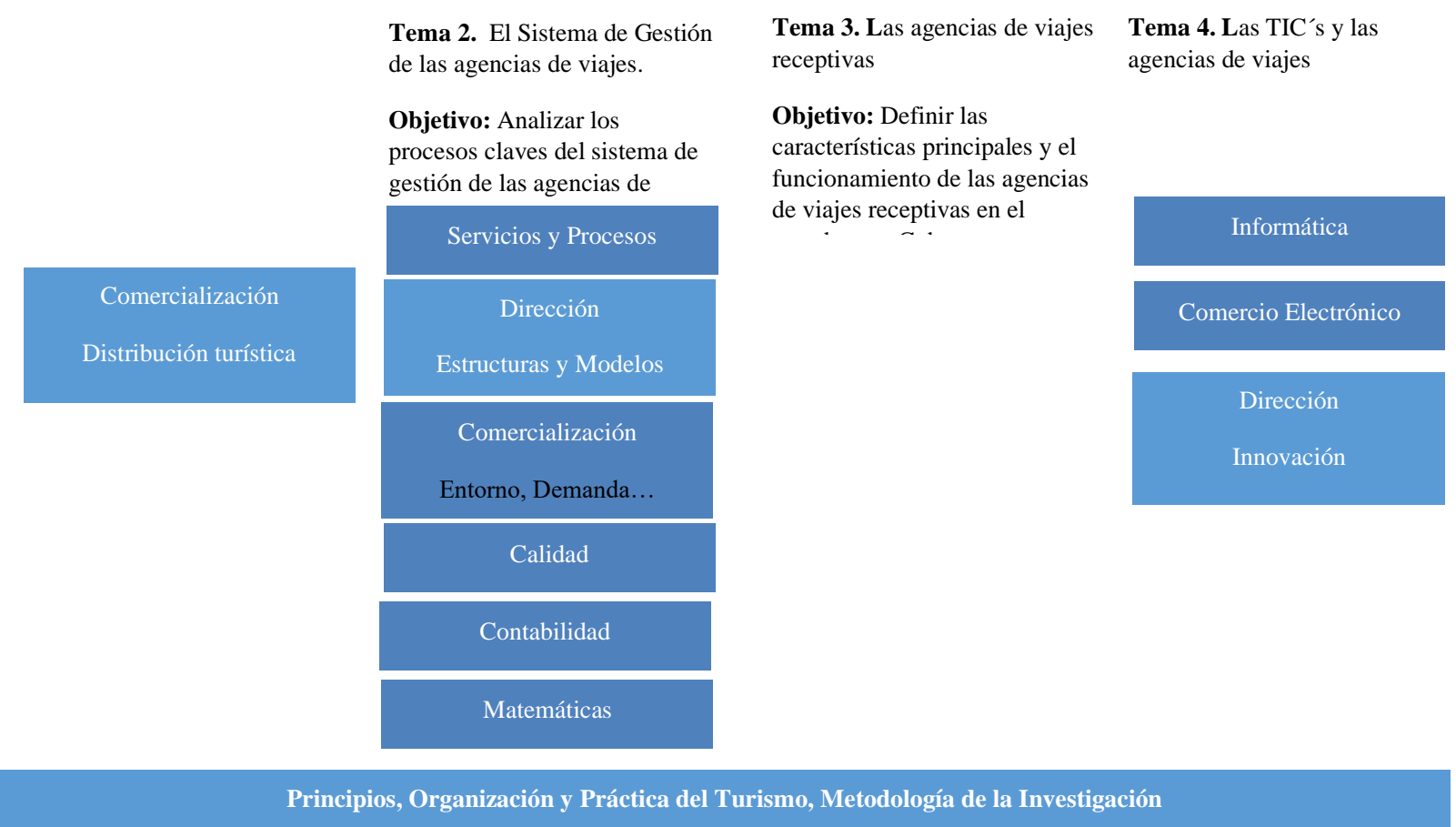

Figura 1. Relación de los temas de Gestión de Agencias de Viaje con otras asignaturas de la carrera Licenciatura en Turismo 


\section{Fuente: Elaboración propia}

No pretende el análisis anterior, responder a todas las necesidades identificadas, pero resulta orientadora tanto para estudiantes, como para profesores. Este análisis, así como el estudio de las tendencias de la actividad de las agencias de viaje en el mundo, de conjunto con entrevistas no estructuradas a profesores de las asignaturas expuestas, indicó, como primer resultado, la necesidad de abordar la innovación en este tipo de empresas como un contenido transversal, toda vez que aparece en todas sus clasificaciones, en la actualidad turística.

A partir de las propias exigencias de la implementación del Plan "E", se requirió de la profundización de las relaciones de la asignatura con la disciplina, para determinar la contribución de la primera al cumplimiento del objetivo de la segunda y en el contexto de la carrera.

Se definieron así:

Tabla 1. Gestión de Agencias de Viajes y relaciones intradisciplinarias.

Nivel de precedencia

Anterior

\section{Asignatura - Relación}

Contribución a Gestión de Agencias de Viajes

Comunicación Interpersonal: Función de venta de las Agencias de Viajes, Redacción en trabajos de curso, Expresión oral en presentaciones.

Gestión de Recursos de Información Turística: Herramienta para la actividad investigativa a evaluar.

Economía Turística: Valoración del macro y microentorno y su incidencia en los viajes.

Gestión de destinos turísticos: El destino como el espacio para el desarrollo de viajes, su planificación o programación.

Relación de su desarrollo con los Turoperadores.

Comercialización: Valoración del Marketing estratégico operativo en las Agencias de Viajes. Tecnologías de la Información y Comunicaciones.

Distribución y Producto. 
Derecho: Marco jurídico vigente en Cuba para la gestión de las AAVV. Incidencia de las generalidades. Ley de Contratación Económica.

Transporte: Características y cotización para programas turísticos.

Posterior

Contribución de Gestión de Agencias de Viajes

Técnicas de Guiar: Las funciones de asistencia. El desempeño del guía como elemento de diferenciación y fuente de ventaja competitiva. Viajes especializados. Definición y características de programas y competencias del guía. Imagen y calidad de las agencias de viaje.

Gestión de eventos ${ }^{[2]}$ : Especialización, doble rol de las agencias de viajes (distribuidoras y organizadoras), Bleisure, actualidad y tendencias.

Relaciones Públicas ${ }^{1}$ : Fam Tryps y Ferias de Turismo como acciones de Relaciones Públicas de las agencias de Viajes. Vínculo con comercialización.

Fuente: Elaboración propia.

Gestión de Agencias de Viajes tributa al cumplimiento de los objetivos de la disciplina Turismo y Viajes (Utilizar métodos y técnicas que apoyan el desarrollo turístico en las diferentes esferas y procesos de la entidad; profundizar en las particularidades de procesos y operaciones y sus manifestaciones en la actividad turística en Cuba y el mundo, así como el enfoque multidisciplinario de su tratamiento científico; dominar los fundamentos necesarios para garantizar una gestión eficiente de la actividad turística a todos los niveles de productos turísticos de viajes) a partir de sus contenidos esenciales relacionados con distribución y desarrollo de destinos turísticos; agencias de viajes y contratación; tendencias de generalización y especialización, integración, concentración y alianzas; programación y cotización y productos, servicios y orientación al cliente.

Tabla 2. Gestión de Agencias de Viajes y relaciones extradisciplinarias

\section{Disciplina Precedencia}

Asignatura - Relación

Integradora Anterior Principios, Organización y Práctica de Turismo:

Agencia de Viajes y sistema turístico. Modelo Pentagonal. 
La Hospitalidad y los Procesos: Filosofía de atención al visitante y servicio de asistencia. Diseño y operaciones como proceso. Diseño de proceso y diseño de programas.

Modalidades Turísticas: Contexto para mantenimiento y ampliación del catálogo de las Agencias de Viaje. Características para viajes especializados.

Investigación Científica en el Turismo: Herramienta, orientación a la demanda, análisis del entorno.

\section{Recursos Turísticos}

\section{Entidades}

Posterior Anterior

Anterior

Dirección y estrategia. El enfoque empresarial de la Agencia de Viajes. Estrategias genéricas y funcionales. Estructura y Relaciones.

Contabilidad Financiera: Nuevo producto e impacto en estados financieros. Ingresos, costos y gastos de las agencias de viajes.

Gestión de Recursos Humanos: Factor de Competitividad.

Gestión de Calidad: Riesgos y Control.

Dirección Integrada de Proyectos: Diseño de Producto, Identificación y valoración de riesgos. Herramental.

Gestión Alojamiento

Gestión Restauración de Prestadores Turísticos. Programación y Diseño de de producto (Cotización, cupos,

Entidades de Ocio

Posterior prestatarios, proveedores, Turoperadores y Agencias de viajes extranjeras. 


\begin{tabular}{lll}
\hline & $\begin{array}{l}\text { Decisiones financieras }{ }^{2} \text { Política crediticia. } \\
\text { Integración, crecimiento y fuentes de financiamiento. }\end{array}$ \\
Matemática & Anterior & Matemática Cálculo e interpretación de datos \\
Aplicada e & Superior & cuantitativos. Análisis de la información \\
Informática & Informática & \\
& Estadística \\
& Matemática \\
& financiera \\
\hline
\end{tabular}

Fuente: Elaboración propia.

El establecimiento de estas relaciones posibilitó la determinación de los contenidos esenciales, y con ello el mejor aprovechamiento de las horas lectivas dedicadas para la asignatura.

\section{Lograr una integración adecuada entre las actividades académicas, laborales e investigativas.}

Es válido destacar que la Licenciatura en Turismo desde su concepción inicial posee un alto componente laboral, y que, a los efectos del presente trabajo, no fue abordado, mientras que en el propio proceso se estableció una dinámica en las actividades académicas e investigativas, utilizando para estas últimas como herramienta las evaluaciones, considerando que "si quiere cambiar los aprendizajes de los estudiantes entonces cambie las formas de evaluar esos aprendizajes" (Brown G., 1997). Se establecen así en la evaluación sistemática dos objetivos:

a) Comprobar conocimientos y habilidades del proceso.

b) Profundizar a través de la investigación en aspectos referidos al entorno nacional e internacional de las agencias de viajes.

Esta aparente división se encuentra interconectada con la primera de las bases conceptuales abordadas, y como se mostrará en el presente trabajo, con aquellas relacionadas a la autogestión del estudiante.

Tributa además a la aplicación de la evaluación como motivación para el aprendizaje y no solo como control de este, o sea como incidente en el comportamiento del estudiante, vinculado al reconocimiento de la necesidad de la investigación en el sector turístico, factor clave de éxito de las empresas y función principal del profesional.

\section{Potenciar el protagonismo del estudiante en su proceso de formación.}

El cumplimiento de este concepto implicó la revisión del tiempo definido para conferencias, seminarios y clases prácticas dentro del curso, si bien, estas últimas forman parte del sistema evaluativo. Total: 56 horas/clases (Seminarios: 18 h/c, Laboratorio: 2 h/c, Clases Prácticas: 16 h/c).

Las apreciaciones anteriores, a partir de la experiencia de las autoras, identificaban a los seminarios como un espacio de evaluación grupal, con las implicaciones de la misma 
(débil diferenciación del desempeño individual), así como la limitación a uno o varios aspectos designados al equipo. Se introduce así la "coevaluación", proceso a través del cual los estudiantes y los profesores participan en la evaluación del trabajo de los estudiantes. Investigadores han encontrado que este tipo de evaluación profundiza la comprensión de los estudiantes de su propio aprendizaje y permite que se involucren de manera más activa y autodirigida en su proceso de aprendizaje (Falchikov, 2005); al definirse para cada equipo el doble rol de ponente y oponente.

La experiencia indicó que en el rol de oponente afloran de forma espontánea integraciones definidas con asignaturas precedentes y pertinentes para cada tema. Las valoraciones críticas grupales en el rol de oponente, contribuyen además a la formación desde el punto de vista profesional del valor "Honestidad" (Brindo siempre una información veraz, soy creíble ante el colectivo") entre otros.

Se introdujo el concepto del seminario problémico, a partir de dinámicas comunicacionales como panel "Presentación de un tema, bajo la coordinación de un moderador para ser analizado y discutido en forma de diálogo o conversación" (Beim, 1998) y mesa redonda "Reunión para actualizar conocimientos, conducida por un moderador que orienta la discusión para que ésta se mantenga en torno al tema principal. Los participantes presentan sus puntos de vista en torno al tema en igualdad de condiciones y con límite de tiempo para posteriormente realizar un debate y responder las preguntas del público" (Colectivo, 2000), "Reunión de expertos que analizan un tema con enfoques divergentes, ante un público de conocedores y especialistas” (Beim, 1998).

Otro aspecto de interés resultó la creación por parte del estudiante, de casos relacionados con la asignatura, en los cuales se muestran la integración de los diferentes temas de la asignatura, el conocimiento de la actualidad turística internacional, creatividad para el diseño de productos e información actualizada de las agencias de viajes nacionales.

\section{Potenciar el tiempo de auto preparación del estudiante.}

Hasta el curso 2019/2020, la asignatura Gestión de agencia de viajes no dispuso de un libro de texto nacional, resulta por tanto este el primero en el cual existe una referencia bibliográfica, elaborada además por un profesor de la Facultad de Turismo de la Universidad de La Habana.

A pesar de la importancia del texto, se requería compilar ejemplos, ejercicios, práctica internacional y orientaciones para los estudiantes; propósito que se cumplió de forma digital, posterior a la definición de las unidades didácticas de cada tema.

De forma general, se presenta tras la Introducción a la asignatura, una estructura estándar que recoge: introducción al tema, exposición de objetivos, explicación de cada unidad didáctica, elaboración de un documento básico, guía para el estudio, orientaciones para la evaluación, guías de autocontrol, ejercitaciones y casos. En cada unidad didáctica, se consideró también la utilización de: cuadros y figuras como recurso para el aprendizaje, recuadros para aspectos importantes, hipervínculos dentro y fuera del documento, 
referencia y materiales para actualizar contenidos de otras asignaturas precedentes que aportan al conocimiento, identificados a partir de las relaciones intra y extradisciplinarias

En el curso 2018 / 2019 en específico, además de la inclusión de bibliografía o documentación en idioma inglés, se insertó en cada tema un miniglosario con vocabulario específico en alemán (segundo idioma).

Esta compilación además está orientada a la enseñanza problémica, con la inclusión en el documento básico de un acápite nominado como "Para pensar", así como la autoevaluación en las "Guías de autocontrol".

La estructura de la compilación, fue valorada por los estudiantes a través de entrevistas no estructuradas, así como por observación y evaluación de los docentes. Los resultados obtenidos fundamentaron su modificación parcial a partir del segundo tema. Se identificó que los estudiantes no gustan de los documentos largos, que muestran preferencias por las presentaciones electrónicas, que no consultan totalmente la bibliografía complementaria, que no completan los casos y que la lectura de las orientaciones de la evaluación es poco activa.

\section{Lograr transformaciones cualitativas en el proceso de formación como} consecuencia de un amplio y generalizado empleo de las Tecnologías de Información y Comunicaciones (TIC's).

Los documentos digitales anteriormente explicados como base material de estudio, autoestudio y orientación, están estructurados para su incorporación a la plataforma interactiva Moodle.

Desde el punto de vista metodológico, el colectivo de la disciplina Turismo y Viajes de la Facultad de Turismo de la Universidad de La Habana, valoró además la posibilidad de utilización total o parcial para el Curso por encuentros y el Curso a distancia. Esta base conceptual se aplica, además, al incorporar al curso tareas con la necesaria navegación en Internet y la clase práctica, con el objetivo de visualizar el uso de las tecnologías por agencias de viaje de cualquier naturaleza, y establecer vínculos entre distribución y comunicación turísticas.

\section{El fortalecimiento de los vínculos de las universidades con los organismos empleadores y todas las instancias que sean fuentes de empleo.}

Las características del Turismo, a partir de su definición como sistema, su utilización intensiva de fuerza de trabajo, la multiplicidad de negocios que engloba y su propia dinámica de desarrollo en el mundo y en el país, exigen la inserción de sus especialistas en el proceso de formación, aún en su componente académico, toda vez que el discurso práctica facilita al estudiante la contrastación de la realidad con el componente científico - técnico del que se apropia en la universidad; mientras que le aporta visibilidad de la solución de problemas, así como experiencias acerca de la identificación y valoración de los mismos.

De tal forma se constituyó para la disciplina y la asignatura un objetivo "Generalizar en el proceso de enseñanza aprendizaje experiencias de la praxis turística cubana". Se 
consideraron, así como clases prácticas, el intercambio con especialistas, en cuanto a los temas de Tecnologías y Representaciones.

Estas actividades propiciaron la creación de un espacio de diálogo, la actualización del entorno empresarial, la información sobre líneas de desarrollo, la capacitación de los profesores, la percepción de aplicabilidad de los conocimientos y la integración en la praxis de conocimientos derivados de múltiples asignaturas.

\section{Lograr transformaciones en la evaluación del aprendizaje.}

Tal y como se ha expuesto en el cuerpo del trabajo, relacionado con otras bases conceptuales, se utilizaron disímiles formas de evaluación, considerando que en las denominadas "tradicionales o convencionales" como la evaluación parcial y la final, se modificaron los enunciados de las preguntas. Vale destacar que "los cambios metodológicos consecuentemente implican cambios en las metodologías de evaluación" (Salinas \& Cotilla, 2007).

Estos autores además plantean que en relación con la evaluación en el aprendizaje profundo "existe una cierta motivación intrínseca basada en el interés por aquello que se trabaja. Hay un interés por comprender más allá del recordar, la información se va "encajando" en esquemas de relación $\mathrm{y}$, además, el conocimiento novedoso va "enlazando" con lo ya conocido. El tipo de conducta que se le demanda al estudiante, en relación con este aprendizaje, es el de comprender, relacionar, construir un discurso propio y relevante, resolución de problemas de forma creativa, etc....", propósitos a los que estuvo orientada esta modificación y, a fin último, la implementación de las bases conceptuales del Plan de estudio E.

Se diseñaron así cuestionarios con respuestas múltiples, definiciones a fundamentar, casos de estudios y la determinación de condicionantes para la toma de decisiones.

\section{Aplicación de cuestionarios a estudiantes. Estructura y resultados.}

A partir del trabajo metodológico del colectivo de la asignatura, se determinaron en primer lugar los ítems a valorar en un primer cuestionario, orientado a la valoración de los documentos de estudio presentados. Se determinaron y definieron:

- Integración: Relación de los contenidos con asignaturas precedentes, que contribuya, por una parte, a la consolidación de las mismas y por otra a la contextualización en el entorno de las agencias de viajes.

- Percepción de aplicabilidad: Valoración, a partir de conocimiento y expectativas del desempeño como futuro profesional, de la utilidad de los documentos para ello.

- Nivel de actualización: Coherencia de los contenidos con el entorno turístico actual y aspectos observables de la gestión de agencias de viajes; relación con la fecha de las publicaciones.

- Nivel de comprensión: Autoevaluación de los estudiantes, de su capacidad para adquirir información y conocimiento a través de la lectura y estudio de los documentos presentados. 
- Utilidad de los materiales: Relacionado con el cumplimiento de los objetivos de la asignatura.

Para todos los cuestionarios aplicados, se utilizó una escala de Lickert de 1 a 5, obteniéndose en el primero de ellos, los siguientes valores:

- El aspecto mejor valorado por los estudiantes es la integración que propicia la compilación. (Promedio 4,5)

- El aspecto con peor evaluación es el nivel de comprensión. (Promedio 3,6)

- Existe una aparente contradicción cuando la percepción de aplicabilidad (Promedio 4,4) resulta muy superior a la valoración de la utilidad de los materiales. (Promedio 3,9)

Se aprecia una amplia dispersión en los valores correspondientes a cada ítem, indicativo de una muestra con diferencias cualitativas individuales, mientras que el coeficiente de correlación entre los ítems valida estadísticamente, el nivel de comprensión como determinante.

Bajo la consideración que las características individuales y las experiencias previas inciden en el comportamiento, se diseñó un segundo instrumento con la estructura siguiente:

Tabla 2. Cuestionario. Experiencias anteriores y motivación.

\begin{tabular}{|c|c|c|c|c|c|}
\hline \multirow[t]{2}{*}{ Pregunta } & \multicolumn{5}{|c|}{ Evaluación } \\
\hline & Siempre & $\begin{array}{l}\text { Casi } \\
\text { Siempre }\end{array}$ & $\begin{array}{l}\text { A } \\
\text { veces }\end{array}$ & $\begin{array}{l}\text { Casi } \\
\text { nunca }\end{array}$ & Nunca \\
\hline \multicolumn{6}{|l|}{$\begin{array}{l}\text { 1. ¿Han dispuesto de orientación de } \\
\text { estudios de materiales previos a clases en } \\
\text { otras asignaturas? }\end{array}$} \\
\hline \multicolumn{6}{|l|}{$\begin{array}{l}\text { 2. ¿Has estudiado los materiales orientados } \\
\text { previos a la clase? }\end{array}$} \\
\hline $\begin{array}{l}\text { 3. ¿Cómo evalúas tu nivel de autogestión } \\
\text { del conocimiento? }\end{array}$ & $\begin{array}{l}\text { Muy } \\
\text { Alto }\end{array}$ & Alto & Medio & Bajo & $\begin{array}{l}\text { Muy } \\
\text { Bajo }\end{array}$ \\
\hline $\begin{array}{l}\text { 4. ¿En qué grado estás motivado a } \\
\text { profundizar en los contenidos? }\end{array}$ & & & & & \\
\hline
\end{tabular}

\section{Fuente: Elaboración propia.}

Para el procesamiento del cuestionario, se ajustó la escala cualitativa a la cuantitativa.

Los resultados obtenidos muestran:

- El promedio de todas las preguntas oscila entre 3 y 3,6 puntos, o sea, medio.

- Los estudiantes no identifican el estudio de materiales previo a la clase como una praxis consolidada en las asignaturas precedentes. (Promedio 3,75).

- El nivel de información referida a la orientación previa de materiales de estudio, es superior al nivel de respuesta de los estudiantes (Pregunta 2, Promedio 3,0) 
- El $62 \%$ de la muestra considera que su nivel de autogestión del conocimiento es alto o muy alto, mientras que solo el $41 \%$ considera su motivación a profundizar de igual forma.

Las diferencias cualitativas individuales de la muestra se ratifican al ser los cuestionamientos respecto al comportamiento $(3,4)$ aquellos con mayores valores de desviación estándar (1,11 y 1,17 respectivamente), lo cual de conjunto con los restantes resultados determinó la necesidad de potenciar el autoestudio y gestión del conocimiento, combinando el control (evaluaciones) y el estímulo al mismo.

Por su parte, el último instrumento, orientado a la valoración de las modificaciones introducidas y evaluación de los objetivos, fue aplicado al cierre del curso y quedó estructurado como se muestra en la tabla a continuación:

Tabla 1. Cuestionario a estudiantes. Valoración de elementos del proceso de enseñanza aprendizaje acorde las modificaciones introducidas.

1. ${ }_{i}$ En qué medida el método empleado ha fomentado el autoestudio y gestión del conocimiento?

2. ¿Qué valor les concede a las actividades con especialistas de la práctica?

3. ¿Considera Ud. adecuada la estructura de la asignatura entre conferencias, clases prácticas y seminarios?

4. ¿Los materiales le resultaron de apoyo suficiente para el conocimiento alcanzado?

5. ¿Como se autoevalúa en cuanto al alcance de los siguientes objetivos?

5.1 explicar principios y conceptos relacionados con las agencias de viajes y entidades de transporte turístico.

5.2 Evaluar la metodología de trabajo con las agencias de viajes y entidades de transporte turístico, así como sus principales características, medios y objetivos de trabajo.

5.3 Aplicar los conocimientos de las principales tendencias identificadas en las agencias de viajes y entidades de transporte turístico para la solución de problemas.

5.4 Analizar la metodología que permita desarrollar las investigaciones turísticas las diferentes direcciones de las actividades correspondientes.

Fuente: Elaboración propia.

El procesamiento de este instrumento, muestra como resultado que:

- Las actividades con especialistas son el aspecto mejor valorado, elemento realzado en la valoración cualitativa. (Promedio 4,6). 
- El método resulta válido para el fomento de la autoestudio y gestión del conocimiento. (Promedio 4,1).

- La suficiencia de los materiales entregados, resulta el aspecto con menor valoración. (Promedio 4,0).

En cuanto a la autovaloración del cumplimiento de los objetivos de la asignatura, el peor evaluado es la solución de problemas, seguido del referido a las investigaciones (Promedio 3,7 y 3,8 respectivamente), lo que se ratifica con el análisis cuantitativo del examen final. Los resultados de ambos objetivos muestran las mayores desviaciones estándar (Ambos 1,1).

Se aprecia, sin embargo, que el nivel de dispersión obtenido con la aplicación de este instrumento resulta inferior al correspondiente al primer cuestionario, de lo que se infiere un impacto positivo de la organización del proceso de enseñanza - aprendizaje, en cuanto a la alineación de la muestra en cuanto a desempeño superior.

De los resultados obtenidos, se resume que:

- Los estudiantes no han incorporado a su comportamiento la autogestión de conocimientos, parte esencial y base conceptual del diseño del Plan "E".

- La tendencia u orientación a la reproductibilidad, existe, aún con el nuevo enfoque metodológico de la asignatura.

- La apropiación de conocimientos de asignaturas anteriores como Dirección, Comercialización y Contabilidad, presenta insuficiencias.

- La inserción de especialistas de la práctica turística cubana fue positiva y altamente valorada por los estudiantes.

\section{Conclusiones}

La demostrada interdependencia de la asignatura Gestión de Agencias de Viajes para el cumplimiento de sus objetivos, exige ampliar y profundizar el trabajo metodológico intra e interdisciplinar de forma sistémica y sistemática, que considere las modificaciones o inclusiones en los planes analíticos de la propia asignatura y las restantes señaladas. Podrá valorarse entonces, la interacción no solo en el trabajo metodológico, sino además en la realización de team teaching.

El propio trabajo metodológico de la asignatura, deberá propiciar, atendiendo a los resultados obtenidos.

- El incremento del número de evaluaciones individuales en detrimento de las colectivas, a partir de su diversificación, entre otras formas.

- El control de forma más efectiva la lectura y estudio de los materiales.

- La revisión de los materiales, evitando duplicidades con el Libro de Texto (disponible a partir del curso 2019-2020)

- La planificación de un mayor número de actividades con especialistas de la práctica. 
- La mayor atención de las diferencias individuales en cada grupo y en función de ello La realización de consultas periódicas, de ser necesario (en especial con los estudiantes extranjeros).

El Plan "E" exige una mayor preparación del profesor, que incorpore, entre otros aspectos, la actualización tanto en el entorno nacional como internacional de la gestión de las agencias de viajes, para su aprovechamiento y conversión en temas de debate o discusión y casos de estudio. (Ejemplo de ello, resultó en el Curso 2019 - 2020, periodo posterior a la presente investigación, la quiebra de Thomas Cook). La visión del Plan "E" implica además una mayor frecuencia y nuevas formas de evaluación, toda vez que, que la autogestión del conocimiento no es un elemento aislado a partir del comportamiento o motivación del estudiante, sino que subyace en las bases conceptuales del mismo.

Los resultados alcanzados no pueden considerarse concluyentes pero las pautas metodológicas definidas son pertinentes.

\section{Referencias bibliográficas}

Beim, C. (1998). El objetivo de nuestra actividad. Congreso de la Confederación de Organizadores de Congresos y Afines de Latinoamérica y el Caribe. (COCAL). Palacio de las Convenciones. La Habana.

Brown G., e. a. (1997). Assessing student learning in Higher Education. London: Routledge.

Colectivo, A. (2000). Glosario de Término de Eventos y afines. Palcograf. La Habana:

Falchikov, N. (2005). Improving Assessment Through Student Involvement: Practical solutions for aiding learning in higher and further education. Routhledge. New York.

Ministerio de Educación Superior. (2016). Plan de Estudio E. Carrera Licenciatura en Turismo. República de Cuba.

Ministerio de Educación Superior. (s.f.). Documento Base para el diseño de los planes de estudio "E”. La Habana, Cuba: http://www.mes.gob.cu.

Salinas \& Cotilla. (2007). La evaluación de los estudiantes en la Educación Superior. Formación PDI. Universidad de Valencia. Servicio de Formación Permanente.

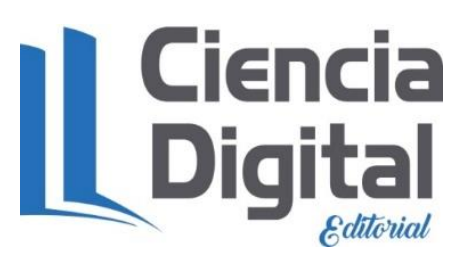




\section{PARA CITAR EL ARTÍCULO INDEXADO.}

Olivera Elosegui, R., \& Rodríguez González, M. (2021). Pautas metodológicas según las bases de planes de estudio "E". Gestión de agencias de viajes. Explorador Digital, 5(1), 281-296. https://doi.org/10.33262/exploradordigital.v5i1.1503

\section{LCiencia}

El artículo que se publica es de exclusiva responsabilidad de los autores y no necesariamente reflejan el pensamiento de la Revista Explorador Digital.

El artículo queda en propiedad de la revista y, por tanto, su publicación parcial y/o total en otro medio tiene que ser autorizado por el director de la Revista Explorador Digital.
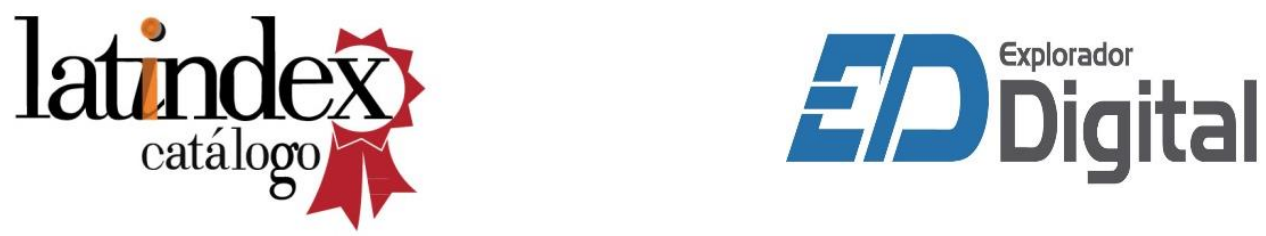